\title{
Synthesis, reactions and antitumor activity of new $\beta$-aminovinyl 3-pyrazolyl ketones
}

\author{
Ahmad Sami Shawali,* Thoraya A. Farghaly and Ahmad R. Al-Dahshoury \\ Department of Chemistry, Faculty of Science, University of Cairo, Giza, Egypt \\ E-mail: as_shawali@mail.com
}

\begin{abstract}
A new series of enaminones 4a-e was prepared and their reaction with the hydrazonoyl chloride 2c gave 3,4'-bis(pyrazolyl)ketones 8a-d. Hydrazinolysis of $\mathbf{8}$ proved to be site selective as it yielded the respective 4-(pyrazol-3-yl)-2H-pyrazolo[3,4- $d]$ pyridazines 9 . The results of screening of the antitumor activity of enaminones 4a-e against human breast cell line MCF-7 revealed that all such compounds exhibited lower activity in relation to the reference drug Doxorubicin and the activity of the enaminone having electron-accepting substituent is more than that having electron donating substituent.
\end{abstract}

Keywords: Hydrazonoyl halides, nitrilimines, hydrazinolysis, enaminones, 1,3-dipolar cycloaddition

\section{Introduction}

Hydrazonoyl halides, $\mathrm{R}-\mathrm{C}(\mathrm{X})=\mathrm{NNHAr}$, are well known versatile precursors for the nitrilimines, $\mathrm{R}-\mathrm{C}\left({ }^{+}\right)=\mathrm{N}-\mathrm{N}\left({ }^{-}\right)$-Ar. Their reactions have been extensively studied and reviewed by one of the authors. ${ }^{1-10}$ Furthermore, it is well known that the $\beta$-enaminones of the general formula $\mathrm{R}_{2} \mathrm{~N}$ $\mathrm{CH}=\mathrm{CH}-\mathrm{COR}$ ' combine the ambident nucleophilicity of enamines with the ambident electrophilicity of enones. ${ }^{11,12}$ Because of this, they have been utilized as versatile valuable precursors for heterocyclic synthesis. ${ }^{13,14}$ Their synthetic applications have been extensively reviewed. ${ }^{12,15-27}$ Furthermore, many enaminones were found to exhibit several biological activities as antitumor, antibacterial and anticonvulsant agents. ${ }^{28,29}$ In the light of these facts, it was thought interesting to synthesize a new series of $\beta$-dimethylaminovinyl 3-pyrazolyl ketones 4 which are novel enaminones that have been unreported hitherto and study their 1,3-dipolar cycloaddition to the nitrilimines, generated in situ by base-catalyzed dehydrohalogenation of hydrazonoyl halides $\mathrm{R}-\mathrm{C}(\mathrm{X})=\mathrm{NNH}-\mathrm{Ar} 2$. Our objectives after such a study are on one hand to screen the antitumor activity of the target enaminones and shed some light on structure activity relationship (SAR) and on the other hand to explore the regiochemistry of the reactions to be 
studied. Herein, we wish to report that such reactions were found to be regiospecific and lead to 3,4'-bis(pyrazolyl)ketones 8 (Scheme 1) which were found useful precursors for synthesis of 4(pyrazol-3-yl)pyrazolo[3,4- $d]$ pyridazines 9 (Scheme 2).

\section{Results and Discussion}

The target new enaminones 4a-e were prepared in this study by refluxing each of the respective pyrazole derivatives $\mathbf{3 a - e ^ { 3 0 , 3 1 }}$ with dimethylformamide-dimethylacetal (DMF-DMA). The structures of the isolated enaminones 4a-e were verified by elemental analyses and spectroscopic methods (IR, ${ }^{1} \mathrm{H}$ NMR and MS) (see Experimental). For example, their ${ }^{1} \mathrm{H}$ NMR spectra displayed, in each case, in addition to the aromatic proton signals, two singlet signals in the regions $\delta$ 2.86-2.93 and 3.13-3.32 characteristic for the $-\mathrm{N}\left(\mathrm{CH}_{3}\right)_{2}$ group, a quartet and triplet signals at $\delta$ 1.06-1.17 and 4.06-4.24 due to the $\mathrm{CH}_{3} \mathrm{CH}_{2} \mathrm{OCO}$ group as well as two doublet signals at $\delta$ 5.62-5.85 and 7.67-7.80 with coupling constant $J=13 \mathrm{~Hz}$ assignable to the two olefinic protons. The latter coupling constant value indicates that the enaminones prepared exist predominantly in the $E$-configuration ${ }^{32}$ depicted in Scheme 1.

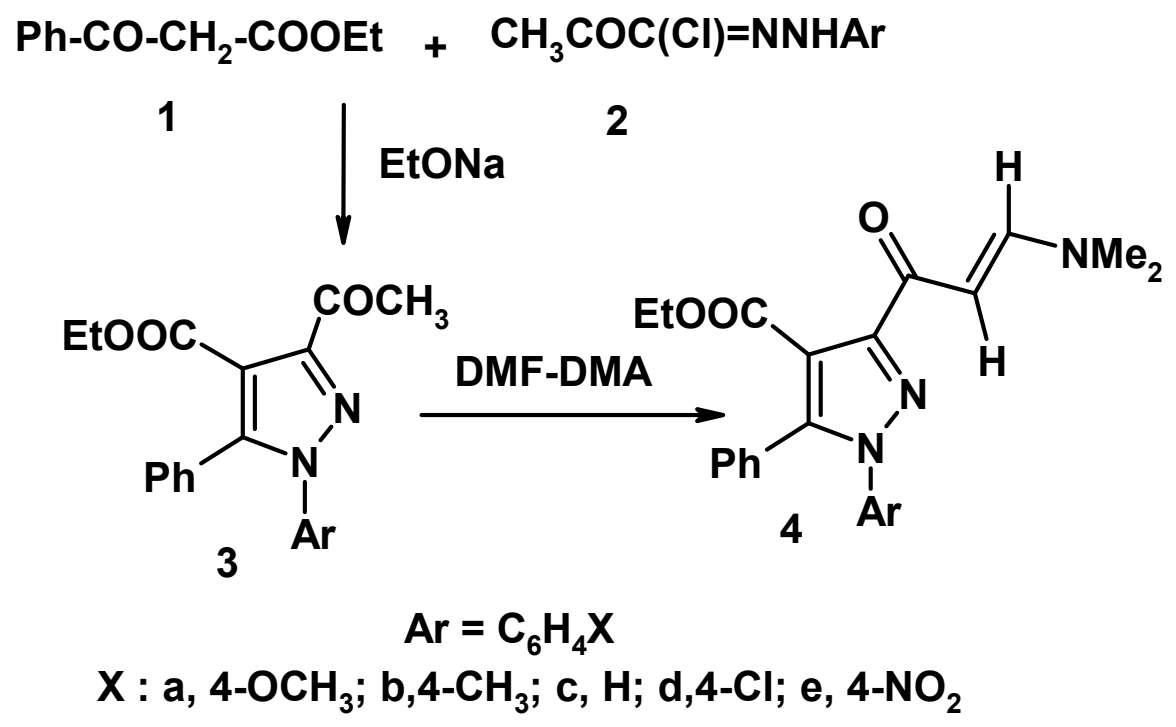

\section{Scheme 1}

Next, the reactions of the enaminones 4a-d, as dipolarophiles, with the nitrilimine generated in situ by dehydrochlorination of $N$-phenyl-2-oxopropanehydrazonoyl chloride 2c were examined. Thus, in our hands, reaction of $\mathbf{4 a - d}$ each with $\mathbf{2 c}$ in refluxing dioxane in the presence of triethylamine yielded, in each case, a single product that was identified, on the basis of its elemental analysis and spectral (IR, ${ }^{1} \mathrm{H}$ NMR and MS) data as 3,4'-bis(pyrazolyl)ketone 8 
(Scheme 2). The other possible isomeric structure namely 3,5'-bis(pyrazolyl)ketone 7 was discarded. For example, the ${ }^{1} \mathrm{H}$ NMR spectra of the products isolated showed in each case a singlet signal in the region $\delta 8.6-9.22$, which corresponds to $\mathrm{H}-5$ of the pyrazole ring residue in the products $\mathbf{8}$. This assignment is based on the fact that in the pyrazole ring system, the C-4 is the most electron rich carbon and so H-4 is expected to be more shielded than H-5 linked to C-5 which is bonded to nitrogen atom. Typically the signal of H-5 usually appears at $\delta 8.66-8.69^{33}$ whereas that of H-4 appears at $\delta 5.81-5.89 .{ }^{34,35}$ Thus, the formation of 8 rather than 7 indicates that the studied reaction of $\mathbf{4}$ with $\mathbf{2}$ is regiospecific. To account for the formation of $\mathbf{8}$, it is suggested that the reaction of $\mathbf{4}$ with $\mathbf{2}$ proceeds via 1,3-dipolar cycloaddition of the nitrilimine, derived from $2 \mathbf{c}$, to the activated double bond in the enaminone 4 to give the respective cycloadduct $\mathbf{6}$ which in turn undergoes in situ elimination of dimethylamine to afford $\mathbf{8}$ as the end product.

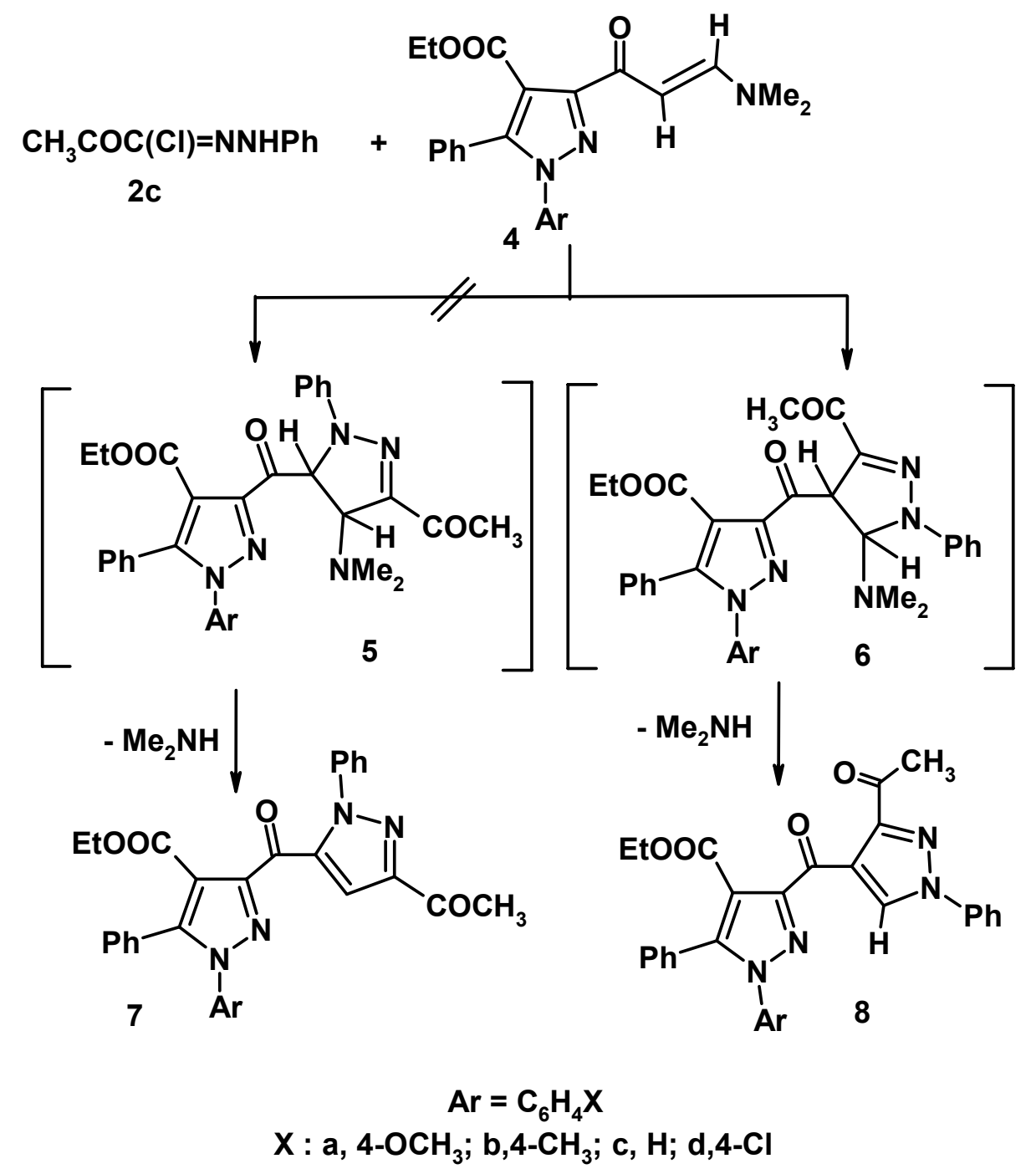

Scheme 2 
Reaction of hydrazine with the products $\mathbf{8}$ was next examined to explore its site selectivity as such a reaction can lead to the production of fused pyrazoles 9 and/or $\mathbf{1 0}$ (Scheme 3). In our hands, treatment of compound $\mathbf{8 a}$ with hydrazine hydrate in refluxing ethanol yielded a product whose spectroscopic (IR, MS, ${ }^{1} \mathrm{H}$ NMR) and elemental analysis data were consistent with structure 9. For example, its mass spectrum showed its molecular ion peak at $\mathrm{m} / \mathrm{z} 530$ and its IR spectrum revealed one carbonyl absorption band at $v 1712 \mathrm{~cm}^{-1}$ assignable to the ester carbonyl group. Its ${ }^{1} \mathrm{H}$ NMR spectrum showed, in addition to the aromatic proton signals, the following signals : $\delta$ 1.06, 4.16, 2,26 and 3.78 assignable to $\mathrm{CH}_{3} \mathrm{CH}_{2} \mathrm{OCO}, \mathrm{CH}_{3}$ and $\mathrm{CH}_{3} \mathrm{O}$ groups.

To provide further evidence for structure of the product 9 , it was compared with an authentic sample of compound $\mathbf{1 0}$ prepared by alternate unambiguous route (Scheme 3). Thus, hydrazinolysis of the pyrazole 3a in refluxing ethanol gave product that was identified as $\mathbf{1 1}$ (Scheme 3) on the basis of its spectra (IR, ${ }^{1} \mathrm{H}$ NMR and MS) and elemental analysis (see Experimental). Reaction of 11 with DMF-DMA gave the enamine 12 which upon treatment with $\mathbf{2 c}$ in dry dioxane in the presence of triethylamine under reflux yielded the product $\mathbf{1 0}$ that was found different from compound 9 isolated from hydrazinolysis of 8a (Scheme 2). The structures of compounds 10, 11 and 12 were elucidated on the basis of their spectra and elemental analyses (see Experimental). For example, in addition to the aromatic proton signals, their ${ }^{1} \mathrm{H}$ NMR spectra revealed the absence of both the triplet and quartet signals of the protons of the $\mathrm{CH}_{3} \mathrm{CH}_{2} \mathrm{OCO}$ group. Compound $\mathbf{1 0}$ exhibits four singlet signals at $\delta 2.54,3.82,8.05$ and 12.0 assignable to the protons of the $\mathrm{CH}_{3} \mathrm{CO}, \mathrm{CH}_{3} \mathrm{O}$, pyrazole- $\mathrm{H}$ and $\mathrm{NHCO}$, respectively. Compound 11 also exhibits three singlet signals at $\delta 2.60,3.83$ and 9.79 due to resonance of the protons of $\mathrm{CH}_{3}, \mathrm{CH}_{3} \mathrm{O}$, and $\mathrm{NHCO}$ groups. Compound 12 exhibits in its ${ }^{1} \mathrm{H}$ NMR four singlet signals at $\delta$ $2.87,3.14,3.29$ and 11.93 in addition two doublet signals at 5.6 and 7.68 assignable to $\left(\mathrm{CH}_{3}\right)_{2} \mathrm{~N}$, $\mathrm{CH}_{3} \mathrm{O}, \mathrm{NH}, \mathrm{N}=\mathrm{C}-\mathrm{CH}=$, $=\mathrm{HC}-\mathrm{N}$, respectively. 


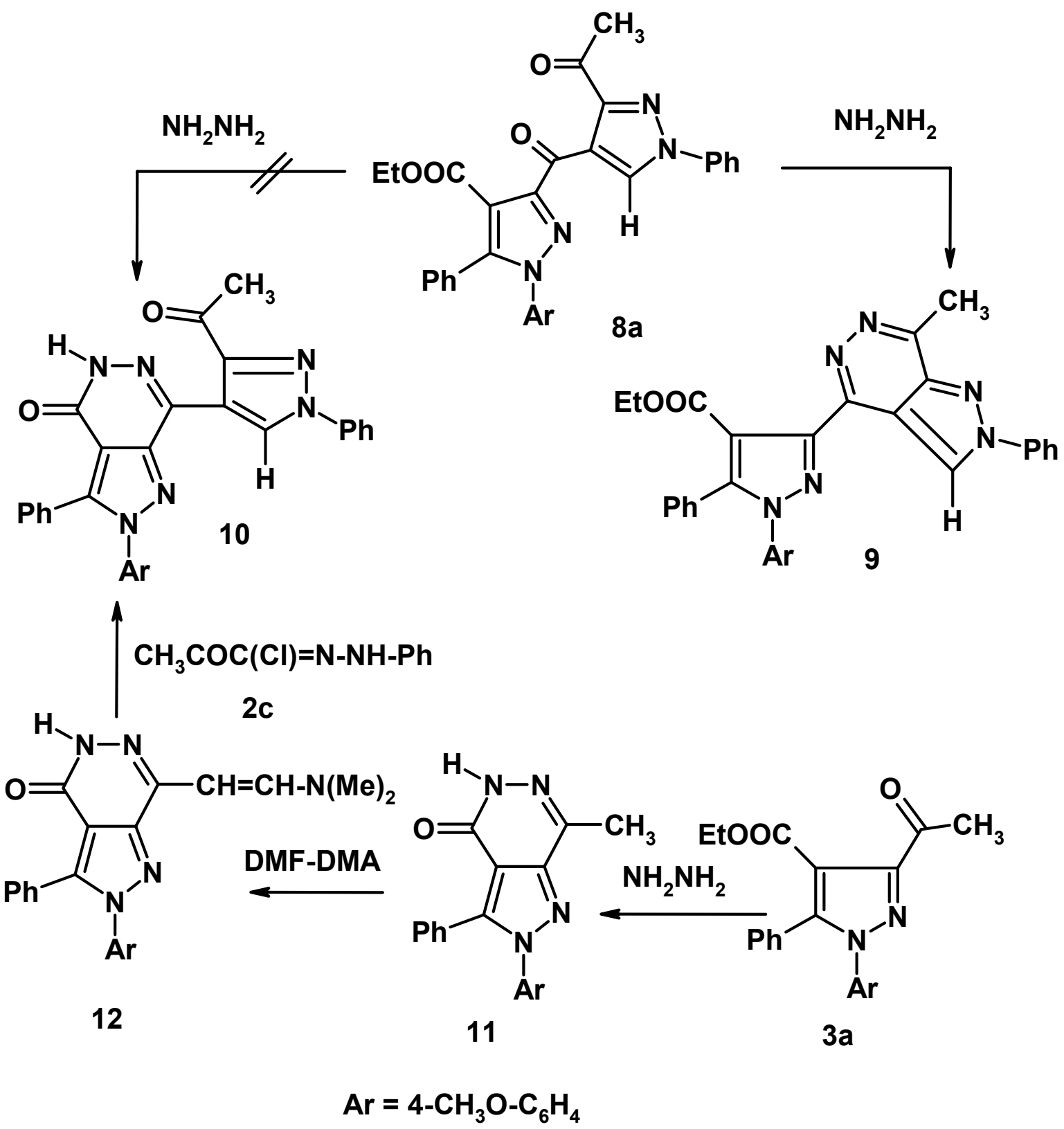

Scheme 3

\section{Antitumor screening and SAR}

The cytotoxic effects of the newly synthesized enaminones 4a-e against human breast cell line MCF-7 were evaluated at the National Institute of Cancer, Cairo, Egypt. Doxorubicin was used as a reference to evaluate the potency of the tested compounds. Five different concentrations of each compound and the reference were used in such screening tests and determination of $\mathrm{IC}_{50}$ values. The results are given in Table 1. As shown in this table, compounds have lower antitumor activity against MCF-7 cell line as their $\mathrm{IC}_{50}$ values are much higher (10.1-11.9) than that of the reference doxotrubicin $\left(\mathrm{IC}_{50}=0.70\right)$. However, the data show that the antitumor 
activity of the studied enaminones $\mathbf{4}$ having electron withdrawing substituents is little bit more than that of those having electron donating ones.

Table 1. In vitro cytotoxic activity of the new compounds 4a-e against MCF-7 cell line

\begin{tabular}{ccccccc}
\hline \multirow{2}{*}{$\begin{array}{c}\text { Compd. } \\
\text { no. }\end{array}$} & $\begin{array}{c}0.0 \\
\mu \mathrm{g} / \mathrm{mL}\end{array}$ & $\begin{array}{c}5.0 \\
\mu \mathrm{g} / \mathrm{mL}\end{array}$ & $\begin{array}{c}12.5 \\
\mu \mathrm{g} / \mathrm{mL}\end{array}$ & $\begin{array}{c}25.0 \\
\mu \mathrm{g} / \mathrm{mL}\end{array}$ & $\begin{array}{c}50.0 \\
\mu \mathrm{g} / \mathrm{mL}\end{array}$ & $\begin{array}{c}\mathrm{IC}_{50} \\
\text { Surviving fraction }\end{array}$ \\
\hline 4a & 1.000 & 0.656 & 0.503 & 0.281 & 0.138 & 11.9 \\
4b & 1.000 & 0.666 & 0.473 & 0.156 & 0.117 & 10.7 \\
4c & 1.000 & 0.680 & 0.441 & 0.194 & 0.118 & 10.3 \\
4d & 1.000 & 0.658 & 0.418 & 0.132 & 0.151 & 10.1 \\
4e & 1.000 & 0.632 & 0.465 & 0.250 & 0.139 & 10.1 \\
Dox. & 1.000 & 0.261 & 0.251 & 0.250 & 0.272 & 0.70 \\
\hline
\end{tabular}

${ }^{\mathrm{a}}$ Doxotrobicin, an antitumor reference.

\section{Conclusion}

In conclusion, a series of new enaminones 4a-e was prepared and their reaction with hydrazonoyl chloride $2 \mathbf{c}$ gave 3,4'-bis(pyrazolyl)ketones 8a-d. Reaction of $\mathbf{8}$ with hydrazine proved to be site selective as it yielded $\mathbf{9}$. The structures of the newly synthesized compounds $\mathbf{4}$, 8-12 were confirmed by spectral data and elemental analyses. The results of screening of the antitumor activity of compounds $4 \mathbf{a}-\mathbf{e}$ revealed that all such compounds exhibited little activity against human breast cell line MCF-7 in relation to the reference drug Doxorubicin and enaminones having electron withdrawing substituents is little bit more than that of those having electron donating substituents. 


\section{Experimental Section}

General. Melting points were determined on an electrothermal Gallenkamp apparatus and are uncorrected. The IR spectra were recorded in $\mathrm{KBr}$ using Pye Unicam SP-1000 Spectrophotometer. ${ }^{1} \mathrm{H}$ NMR spectra were recorded in $\mathrm{CDCl}_{3}$ and DMSO- $\mathrm{d}_{6}$ using a Varian EM$300 \mathrm{MHz}$ Spectrometer, and TMS as internal reference. Mass spectra were recorded on AEI MS 30 mass spectrometer operating at $70 \mathrm{eV}$. Elemental analyses were carried out by the Microanalytical Center of Cairo University, Giza, Egypt. Hydrazonoyl halides 2a-e were prepared following literature procedures. ${ }^{36}$

Ethyl 3-acetyl-1-aryl-5-phenyl-1H-pyrazole-4-carboxylates (3a-e). General method: To sodium ethoxide solution, [prepared from sodium metal $(0.06 \mathrm{~g}, 2.5 \mathrm{mmole})$ and absolute ethanol $(15 \mathrm{~mL})]$ was added ethyl benzoylacetate $1(0.48 \mathrm{~g}, 2.5 \mathrm{mmole})$. The mixture was stirred for 10 min. To the resulting solution was added the appropriate hydrazonoyl chloride 2 ( 2.5 mmole of each) and the reaction mixture was left over night at room temperature, while being stirred. The solid, that precipitated, was filtered off, washed with water, dried and finally crystallized from the appropriate solvent to give the respective 3. The compounds 3a-e prepared together with their physical constants are listed below.

Ethyl 3-acetyl-1-(4-methoxyphenyl)-5-phenyl-1H-pyrazole-4-carboxylate (3a). Yellow crystals, $\left(82 \%\right.$ yield), mp: $106^{\circ} \mathrm{C}(\mathrm{EtOH})$; IR $(\mathrm{KBr}) v_{\max } / \mathrm{cm}^{-1} 1724,1696(2 \mathrm{C}=\mathrm{O}) ;{ }^{1} \mathrm{H}$ NMR $\left(\mathrm{CDCl}_{3}\right): \delta 1.21\left(\mathrm{t}, J=7 \mathrm{~Hz}, 3 \mathrm{H}, \mathrm{CH}_{3}\right), 2.67\left(\mathrm{~s}, 3 \mathrm{H}, \mathrm{COCH}_{3}\right), 3.80\left(\mathrm{~s}, 3 \mathrm{H}, \mathrm{OCH}_{3}\right), 4.27$ (q, $J=$ $\left.7 \mathrm{~Hz}, 2 \mathrm{H}, \mathrm{CH}_{2}\right), 6.82(\mathrm{~d}, J=9 \mathrm{~Hz}, 2 \mathrm{H}, \mathrm{ArH}), 7.15$ (d, $\left.J=9 \mathrm{~Hz}, 2 \mathrm{H}, \mathrm{Ar}-\mathrm{H}\right), 7.27-7.35$ (m, 5H ArH); MS m/z (\%): $365\left(\mathrm{M}^{+}+1,40\right), 364\left(\mathrm{M}^{+}, 100\right), 319$ (64), 277 (20), 210 (79), 77 (77). Anal. Calcd. for $\mathrm{C}_{21} \mathrm{H}_{20} \mathrm{~N}_{2} \mathrm{O}_{4}$ (364.40): C, 69.22; H, 5.53; N, 7.69. Found: C, 69.08; H, 5.39; N, 7.58\%.

Ethyl 3-acetyl-1-(4-methylphenyl)-5-phenyl-1H-pyrazole-4-carboxylate (3b). Yellow crystals, $(84 \%$ yield $), \mathrm{mp} 100^{\circ} \mathrm{C}(\mathrm{EtOH})\left(\text { Lit. mp. } 112^{\circ} \mathrm{C}\right)^{30}$; IR $(\mathrm{KBr}) v_{\max } / \mathrm{cm}^{-1} 1721,1689$ $(2 \mathrm{C}=\mathrm{O}) ;{ }^{1} \mathrm{H}$ NMR $\left(\mathrm{CDCl}_{3}\right): \delta 1.20\left(\mathrm{t}, J=7 \mathrm{~Hz}, 3 \mathrm{H}, \mathrm{CH}_{3}\right), 2.35\left(\mathrm{~s}, 3 \mathrm{H}, \mathrm{CH}_{3}\right), 2.67(\mathrm{~s}, 3 \mathrm{H}$, $\mathrm{COCH}_{3}$ ), 4.23 (q, $\left.J=7 \mathrm{~Hz}, 2 \mathrm{H}, \mathrm{CH}_{2}\right), 7.13-7.40(\mathrm{~m}, 9 \mathrm{H}, \mathrm{Ar}-\mathrm{H}) ; \mathrm{MS} \mathrm{m} / \mathrm{z}(\%): 349\left(\mathrm{M}^{+}+1,14\right)$, 348 ( $\left.\mathrm{M}^{+}, 63\right), 333$ (47), 303 (74), 261 (29), 194 (71), 105 (13), 91 (100), 77 (35). Anal. Calcd. for $\mathrm{C}_{21} \mathrm{H}_{20} \mathrm{~N}_{2} \mathrm{O}_{3}$ (348.41): C, 72.40; H, 5.79; N, 8.04. Found: C, 72.51; H, 5.84; N, 8.16\%.

Ethyl 3-acetyl-1,5-diphenyl-1H-pyrazole-4-carboxylate (3c). Yellow crystals, (85\% yield), mp $88^{\circ} \mathrm{C}(\mathrm{EtOH})$, (Lit. mp. $\left.88^{\circ} \mathrm{C}\right) .^{31}$

Ethyl 3-acetyl-1-(4-chlorophenyl)-5-phenyl-1H-pyrazole-4-carboxylate (3d). Yellow crystals, (86\% yield), mp $114-116^{\circ} \mathrm{C}(\mathrm{EtOH})\left(\right.$ Lit. mp. $\left.116^{\circ} \mathrm{C}\right) .^{30}$

Ethyl 3-acetyl-1-(4-nitrophenyl)-5-phenyl-1 $\mathrm{H}$-pyrazole-4-carboxylate (3e). Pale red crystals, (80\% yield), mp $118^{\circ} \mathrm{C}(\mathrm{MeOH}) ; \mathrm{IR}(\mathrm{KBr}) v_{\max } / \mathrm{cm}^{-1} 1725,1689(2 \mathrm{C}=\mathrm{O}) ;{ }^{1} \mathrm{H} \mathrm{NMR}\left(\mathrm{CDCl}_{3}\right): \delta$ 1.09 (t, $\left.J=7 \mathrm{~Hz}, 3 \mathrm{H}, \mathrm{CH}_{3}\right), 2.61\left(\mathrm{~s}, 3 \mathrm{H}, \mathrm{COCH}_{3}\right), 4.14\left(\mathrm{q}, J=7 \mathrm{~Hz}, 2 \mathrm{H}, \mathrm{CH}_{2}\right), 7.31-7.44(\mathrm{~m}, 5 \mathrm{H}$ Ar-H), 7.59 (d, $J=9 H z, 2 \mathrm{H}, \mathrm{Ar}-\mathrm{H}), 8.25$ (d, $J=9 H z, 2 \mathrm{H}, \mathrm{Ar}-\mathrm{H}) ; \mathrm{MS} \mathrm{m} / \mathrm{z}(\%): 380\left(\mathrm{M}^{+}+1,12\right)$, $379\left(\mathrm{M}^{+}, 38\right), 378$ (11), 349 (16), 288 (24), 179 (58), 90 (10), 76 (100). Anal. Calcd. for $\mathrm{C}_{20} \mathrm{H}_{17} \mathrm{~N}_{3} \mathrm{O}_{5}$ (379.38): C, 63.32; H, 4.52; N, 11.08. Found: C, 63.25; H, 4.39; N, 10.96\%. 
Ethyl 3-[E-3-( $N, N$-dimethylamino)acryloyl]-1-aryl-5-phenyl-1 $H$-pyrazole-4-carboxylate (4a-e). General method: A mixture of the appropriate pyrazole derivative 3 (10 mmol of each) and dimethylformamide-dimethylacetal (DMF-DMA) $(2.4 \mathrm{~g}, 20 \mathrm{mmol})$ was refluxed for 20-30 h then left to cool. Methanol was added to the cold mixture. The resulting solid was collected by filtration, washed with methanol, dried and finally crystallized from ethanol to afford the respective enaminone 4 . The compounds 4 a-e together with their physical constants are listed below.

Ethyl 3-[E-3-( $N, N$-dimethylamino)acryloyl]-1-(4-methoxyphenyl)-5-phenyl-1 $H$-pyrazole-4carboxylate (4a). Brown crystal, $\left(80 \%\right.$ yield), mp $186^{\circ} \mathrm{C}$; IR $(\mathrm{KBr}) v_{\max } 1730,1643(2 \mathrm{C}=\mathrm{O}) \mathrm{cm}^{-}$ ${ }^{1} ;{ }^{1} \mathrm{H}$ NMR $\left(\mathrm{CDCl}_{3}\right): \delta 1.17\left(\mathrm{t}, J=7 \mathrm{~Hz}, 3 \mathrm{H}, \mathrm{CH}_{3}\right), 2.92\left(\mathrm{~s}, 6 \mathrm{H}, \mathrm{CH}_{3}\right), 3.11\left(\mathrm{~s}, 3 \mathrm{H}, \mathrm{OCH}_{3}\right), 4.24$ (q, $\left.J=7 \mathrm{~Hz}, 2 \mathrm{H}, \mathrm{CH}_{2}\right), 5.85(\mathrm{~d}, J=13 \mathrm{~Hz}, 1 \mathrm{H}, \mathrm{CO}-\mathrm{CH}=), 6.78(\mathrm{~d}, J=9 \mathrm{~Hz}, 2 \mathrm{H}, \mathrm{Ar}-\mathrm{H}), 7.14(\mathrm{~d}, J=$ $9 \mathrm{~Hz}, 2 \mathrm{H}, \mathrm{Ar}-\mathrm{H}), 7.25-7.33$ (m, 5H, Ar-H), 7.80 (d, J=13Hz, 1H, =CH-N-); MS m/z (\%): 420 $\left(\mathrm{M}^{+}+1,11\right), 419\left(\mathrm{M}^{+}, 18\right), 405$ (19), 358 (17), 356 (49), 331 (26), 210 (59), 134 (19), 98 (100), 77 (30). Anal. Calcd. for $\mathrm{C}_{24} \mathrm{H}_{25} \mathrm{~N}_{3} \mathrm{O}_{4}$ (419.48): C, 68.72; H, 6.01; N, 10.02. Found: C, 68.66; H, $6.12 ; \mathrm{N}, 10.16 \%$.

Ethyl 3-[E-3-(N,N-dimethylamino)acryloyl]-1-(4-methylphenyl)-5-phenyl-1 H-pyrazole-4carboxylate (4b). Yellow crystals, $\left(82 \%\right.$ yield), $\mathrm{mp} 174^{\circ} \mathrm{C}$; IR $(\mathrm{KBr}) v_{\max } 1715,1649(2 \mathrm{C}=\mathrm{O})$ $\mathrm{cm}^{-1}$; ${ }^{1} \mathrm{H}$ NMR (DMSO-d 6 ) $\delta 1.07\left(\mathrm{t}, J=7 \mathrm{~Hz}, 3 \mathrm{H}, \mathrm{CH}_{3}\right), 2.30\left(\mathrm{~s}, 3 \mathrm{H}, \mathrm{CH}_{3}\right), 2.86\left(\mathrm{~s}, 3 \mathrm{H}, \mathrm{CH}_{3}\right)$, 3.14 (s, 3H, $\left.\mathrm{CH}_{3}\right), 4.09$ (q, $\left.J=7 \mathrm{~Hz}, 2 \mathrm{H}, \mathrm{CH}_{2}\right), 5.64$ (d, $\left.J=13 \mathrm{~Hz}, 1 \mathrm{H}, \mathrm{CO}-\mathrm{CH}=\right)$, 7.18-7.38 (m, 9H, Ar-H), 7.67 (d, J=13Hz, 1H, =CH-N-); MS m/z (\%): $404\left(\mathrm{M}^{+}+1,1\right), 403\left(\mathrm{M}^{+}, 30\right), 386(41)$, 340 (88), 312 (22), 194 (38), 98 (95), 84 (44), 70 (100). Anal. Calcd. for $\mathrm{C}_{24} \mathrm{H}_{25} \mathrm{~N}_{3} \mathrm{O}_{3}(403.49)$ : C, 71.44; H, 6.25; N, 10.41. Found: C, 71.28; H, 6.18; N, 10.37\%.

Ethyl 3-[E-3-(N,N-dimethylamino)acryloyl]-1,5-diphenyl-1 $H$-pyrazole-4-carboxylate (4c). Yellow crystals, $\left(75 \%\right.$ yield), mp $140^{\circ} \mathrm{C}$; IR $(\mathrm{KBr}) v_{\max } 1728,1645(2 \mathrm{C}=\mathrm{O}) \mathrm{cm}^{-1} ;{ }^{1} \mathrm{H}$ NMR $\left(\mathrm{DMSO}-d_{6}\right) \delta 1.08\left(\mathrm{t}, J=7 \mathrm{~Hz}, 3 \mathrm{H}, \mathrm{CH}_{3}\right), 2.86\left(\mathrm{~s}, 3 \mathrm{H}, \mathrm{CH}_{3}\right), 3.32\left(\mathrm{~s}, 3 \mathrm{H}, \mathrm{CH}_{3}\right), 4.09$ (q, $J=7 \mathrm{~Hz}$, $\left.2 \mathrm{H}, \mathrm{CH}_{2}\right), 5.62$ (d, $\left.J=13 \mathrm{~Hz}, 1 \mathrm{H}, \mathrm{CO}-\mathrm{CH}=\right)$, 7.24-7.40 (m, 10H, Ar-H), 7.69 (d, $J=13 \mathrm{~Hz}, 1 \mathrm{H}$, $=\mathrm{CH}-\mathrm{N}-)$; MS m/z (\%): $390\left(\mathrm{M}^{+}+1,3\right), 389\left(\mathrm{M}^{+}, 5\right), 326$ (27), 98 (100), 82 (25), 77 (95). Anal. Calcd. for $\mathrm{C}_{23} \mathrm{H}_{23} \mathrm{~N}_{3} \mathrm{O}_{3}$ (389.46): C, 70.93; H, 5.95; N, 10.79. Found: C, 70.78; H, 5.84; N, $10.66 \%$.

Ethyl 3-[E-3-( $N, N$-dimethylamino)acryloyl]-1-(4-chlorophenyl)-5-phenyl-1 $H$-pyrazole-4carboxylate (4d). Yellow crystals, $\left(80 \%\right.$ yield), $\mathrm{mp} 180^{\circ} \mathrm{C}$; IR $(\mathrm{KBr}) v_{\max } 1732,1641(2 \mathrm{C}=\mathrm{O})$ $\mathrm{cm}^{-1} ;{ }^{1} \mathrm{H}$ NMR $\left(\mathrm{CDCl}_{3}\right): \delta 1.17\left(\mathrm{t}, J=7 \mathrm{~Hz}, 3 \mathrm{H}, \mathrm{CH}_{3}\right), 2.93\left(\mathrm{~s}, 3 \mathrm{H}, \mathrm{CH}_{3}\right), 3.13\left(\mathrm{~s}, 3 \mathrm{H}, \mathrm{CH}_{3}\right), 4.21$ (q, $\left.J=7 H z, 2 \mathrm{H}, \mathrm{CH}_{2}\right), 5.82(\mathrm{~d}, J=13 \mathrm{~Hz}, 1 \mathrm{H}, \mathrm{CO}-\mathrm{CH}=), 7.17$ (d, $\left.J=8 H z, 2 \mathrm{H}, \mathrm{Ar}-\mathrm{H}\right), 7.20-7.33$ (m, 5H, Ar-H), 7.35 (d, J=8Hz, 2H, Ar-H), 7.80 (d, J=13Hz, 1H, =CH-N-); MS m/z (\%): 426 $\left(\mathrm{M}^{+}+2,10\right), 425\left(\mathrm{M}^{+}+1,4\right), 424\left(\mathrm{M}^{+}, 5\right), 423$ (25), 394 (11), 379 (11), 308 (18), 281 (14), 175 (12), 111 (30), 98 (66), 84 (44), 77 (15). Anal. Calcd. for $\mathrm{C}_{23} \mathrm{H}_{22} \mathrm{ClN}_{3} \mathrm{O}_{3}$ (423.90): C, 65.17; $\mathrm{H}$, 5.23; N, 9.91. Found: C, 65.09; H, 5.33; N, 9.87\%.

Ethyl 3-[E-3-( $N, \boldsymbol{N}$-dimethylamino)acryloyl]-1-(4-nitrophenyl)-5-phenyl-1 $H$-pyrazole-4carboxylate (4e). Red crystals, $\left(85 \%\right.$ yield), mp $196^{\circ} \mathrm{C}$; IR $(\mathrm{KBr}) v_{\max } 1730,1644(2 \mathrm{C}=\mathrm{O}) \mathrm{cm}^{-1}$; ${ }^{1} \mathrm{H}$ NMR $\left(\mathrm{DMSO}_{6}\right) \delta 1.06\left(\mathrm{t}, J=7 \mathrm{~Hz}, 3 \mathrm{H}, \mathrm{CH}_{3}\right), 2.88\left(\mathrm{~s}, 3 \mathrm{H}, \mathrm{CH}_{3}\right), 3.15\left(\mathrm{~s}, 3 \mathrm{H}, \mathrm{CH}_{3}\right), 4.06$ (q, 
$\left.J=7 \mathrm{~Hz}, 2 \mathrm{H}, \mathrm{CH}_{2}\right), 5.64(\mathrm{~d}, J=13 \mathrm{~Hz}, 1 \mathrm{H}, \mathrm{CO}-\mathrm{CH}=), 7.31-7.53(\mathrm{~m}, 5 \mathrm{H}, \mathrm{Ar}-\mathrm{H}), 7.55(\mathrm{~d}, J=9 \mathrm{~Hz}$, 2H, ArH), $7.71(\mathrm{~d}, J=9 \mathrm{~Hz}, 2 \mathrm{H}, \mathrm{ArH}), 8.21(\mathrm{~d}, J=13 \mathrm{~Hz}, 1 \mathrm{H},=\mathrm{CH}-\mathrm{N}-)$; MS m/z (\%): 435 $\left(\mathrm{M}^{+}+1,2\right), 434\left(\mathrm{M}^{+}, 5\right), 371$ (69), 179 (10), 98 (100), 76 (23). Anal. Calcd. for $\mathrm{C}_{23} \mathrm{H}_{22} \mathrm{~N}_{4} \mathrm{O}_{5}$ (434.46): C, 63.59; H, 5.10; N, 12.90. Found: C, 63.44; H, 4.99; N, 12.79\%.

Ethyl 3-[(3-acetyl-1-phenyl-1H-4-pyrazolyl)carbonyl]-1-aryl-5-phenyl-1H-pyrazole-4carboxylate (8a-e). General procedure - To a stirred solution of the appropriate enaminone 4 (1 mmol of each) and the hydrazonoyl chloride $2 \mathbf{a}(0.196 \mathrm{~g}, 1 \mathrm{mmol})$ in dry dioxane $(30 \mathrm{~mL})$, triethylamine $(0.5 \mathrm{~mL})$ was added and the mixture was refluxed for $20 \mathrm{~h}$. The precipitated triethylamine hydrochloride was filtered off, and the filtrate was evaporated under reduced pressure. The residue was triturated with methanol. The solid product, so formed in each case, was collected by filtration, washed with water, dried, and crystallized from ethanol to afford the corresponding pyrazole derivatives 8. The compounds 8a-d prepared are listed below together with their physical constants.

Ethyl 3-[(3-acetyl-1-phenyl-1H-4-pyrazolyl)carbonyl]-1-(4-methoxyphenyl)-5-phenyl-1Hpyrazole-4-carboxylate (8a). Brown solid, (70\% yield), mp $158{ }^{\circ} \mathrm{C}$; IR (KBr) $v_{\max } 1727,1670$, $1642(\mathrm{C}=\mathrm{O}) \mathrm{cm}^{-1} ;{ }^{1} \mathrm{H}$ NMR (DMSO-d 6$) \delta 1.16\left(\mathrm{t}, J=7 \mathrm{~Hz}, 3 \mathrm{H}, \mathrm{CH}_{3}\right), 2.68\left(\mathrm{~s}, 3 \mathrm{H}, \mathrm{COCH}_{3}\right), 3.77$ (s, 3H, $\left.\mathrm{OCH}_{3}\right), 4.25$ (q, $\left.J=7 \mathrm{~Hz}, 2 \mathrm{H}, \mathrm{CH}_{2}\right), 6.79-7.80$ (m, 14H, Ar-H), 8.60 (s, 1H, pyrazole); MS m/z (\%) $535\left(\mathrm{M}^{+}+1,1\right), 534\left(\mathrm{M}^{+}, 1\right), 356$ (35), 210 (16), 98 (100), 77 (18). Anal. Calcd. for $\mathrm{C}_{31} \mathrm{H}_{26} \mathrm{~N}_{4} \mathrm{O}_{5}$ (534.58): C, 69.65; H, 4.90; N, 10.48. Found: C, 69.43 ; H, 4.99 ; N, 10.68\%.

Ethyl 3-[(3-acetyl-1-phenyl-1H-4-pyrazolyl)carbonyl]-1-(4-methylphenyl)-5-phenyl-1Hpyrazole-4-carboxylate (8b). Yellow solid, (68\% yield), mp $146{ }^{\circ} \mathrm{C}$; IR (KBr) $v_{\max } 1720,1666$, $1649(\mathrm{C}=\mathrm{O}) \mathrm{cm}^{-1} ;{ }^{1} \mathrm{H}$ NMR (DMSO-d 6$) \delta 1.15\left(\mathrm{t}, J=7 \mathrm{~Hz}, 3 \mathrm{H}, \mathrm{CH}_{3}\right), 2.33\left(\mathrm{~s}, 3 \mathrm{H}, \mathrm{CH}_{3}\right), 2.68(\mathrm{~s}$, $3 \mathrm{H}, \mathrm{COCH}_{3}$ ), 4.20 (q, $J=7 \mathrm{~Hz}, 2 \mathrm{H}, \mathrm{CH}_{2}$ ), 6.98-7.77 (m, 14H, Ar-H), 8.60 (s, 1H, pyrazole H); MS m/z (\%) $519\left(\mathrm{M}^{+}+1,5\right), 518\left(\mathrm{M}^{+}, 8\right), 211$ (100), 154 (38), 104 (24), 77 (19). Anal. Calcd. for $\mathrm{C}_{31} \mathrm{H}_{26} \mathrm{~N}_{4} \mathrm{O}_{4}$ (518.58): C, 71.80; H, 5.05; N, 10.80. Found: C, 71.80; H, 5.51; N, $11.13 \%$.

Ethyl 3-[(3-acetyl-1-phenyl-1H-4-pyrazolyl)carbonyl]-1,5-diphenyl-1 H-pyrazole-4carboxylate (8c). Yellow solid, (75\% yield), mp $110{ }^{\circ} \mathrm{C}$; IR (KBr) $v_{\max } 1723,1700,1655$ $(3 \mathrm{C}=\mathrm{O}) \mathrm{cm}^{-1} ;{ }^{1} \mathrm{H}$ NMR (DMSO-d $) \delta 1.05\left(\mathrm{t}, J=7 \mathrm{~Hz}, 3 \mathrm{H}, \mathrm{CH}_{3}\right), 2.57$ (s, 3H, $\left.\mathrm{COCH}_{3}\right), 4.09$ (q, $J$ $\left.=7 \mathrm{~Hz}, 2 \mathrm{H}, \mathrm{CH}_{2}\right), 7.29-7.99(\mathrm{~m}, 15 \mathrm{H}, \mathrm{Ar}-\mathrm{H}), 9.22(\mathrm{~s}, 1 \mathrm{H}$, pyrazole $\mathrm{H}) ; \mathrm{MS} \mathrm{m} / \mathrm{z}(\%) 505\left(\mathrm{M}^{+}+1\right.$, 6), $504\left(\mathrm{M}^{+}, 20\right), 489$ (12), 213 (13), 180 (25), 104 (16), 77 (100). Anal. Calcd. for $\mathrm{C}_{30} \mathrm{H}_{24} \mathrm{~N}_{4} \mathrm{O}_{4}$ (504.55): C, 71.42; H, 4.79; N, 11.10. Found: C, 71.29; H, 4.88; N, 11.24\%.

Ethyl 3-[(3-acetyl-1-phenyl-1 H-4-pyrazolyl)carbonyl]-1-(4-chlorophenyl)-5-phenyl-1Hpyrazole-4-carboxylate (8d). Yellow solid, (75\% yield), mp $130{ }^{\circ} \mathrm{C}$; IR $(\mathrm{KBr}) v_{\max } 1730,1700$, $1644(3 \mathrm{C}=\mathrm{O}) \mathrm{cm}^{-1} ;{ }^{1} \mathrm{H}$ NMR $\left(\mathrm{DMSO}-d_{6}\right) \delta 1.15\left(\mathrm{t}, J=7 \mathrm{~Hz}, 3 \mathrm{H}, \mathrm{CH}_{3}\right), 2.67\left(\mathrm{~s}, 3 \mathrm{H}, \mathrm{COCH}_{3}\right)$, 4.25 (q, $\left.J=7 H z, 2 \mathrm{H}, \mathrm{CH}_{2}\right), 7.12-7.81(\mathrm{~m}, 14 \mathrm{H}, \mathrm{Ar}-\mathrm{H}), 8.58(\mathrm{~s}, 1 \mathrm{H}$, pyrazole H); MS m/z (\%) $540\left(\mathrm{M}^{+}+2,6\right), 539\left(\mathrm{M}^{+}+1,9\right), 538\left(\mathrm{M}^{+}, 20\right), 360$ (29), 214 (21), 98 (100), 77 (38). Anal. Calcd. for $\mathrm{C}_{30} \mathrm{H}_{23} \mathrm{ClN}_{4} \mathrm{O}_{4}$ (538.00): C, 66.85; H, 4.30; N, 10.39. Found: C, 66.72; H, 4.19; N, 10.28\%. 


\section{Synthesis of compounds 9 and 11}

A mixture of compound 8a $(5 \mathrm{mmole})$ and hydrazine hydrate $(5 \mathrm{~mL})$ in absolute ethanol was refluxed for $10 \mathrm{~h}$ and the reaction mixture was cold. The solid that precipitated was filtered off and crystallized from ethanol to give compound 9.

When the above procedure was repeated using compound 3a in place of $\mathbf{8 a}$, it yielded the respective pyrazolo[3,4- $d$ ] pyridazine 11. The compounds 9 and $\mathbf{1 1}$ are listed below together with their physical constants.

4-[4-Ethoxycarbonyl-1-(4-methoxyphenyl)-5-phenyl-pyrazol-3-yl)]-7-methyl-2-phenyl-2Hpyrazolo[3,4-d]pyridazine (9). Yellow solid, (65\% yield), mp $160{ }^{\circ} \mathrm{C}$; IR (KBr) $\lambda_{\max } 1712$ $(\mathrm{C}=\mathrm{O}) \mathrm{cm}^{-1} ;{ }^{1} \mathrm{H} \mathrm{NMR}\left(\mathrm{CDCl}_{3}\right) \delta 1.06\left(\mathrm{t}, J=7 \mathrm{~Hz}, 3 \mathrm{H}, \mathrm{CH}_{3}\right), 2.26\left(\mathrm{~s}, 3 \mathrm{H}, \mathrm{CH}_{3}\right), 3.78(\mathrm{~s}, 3 \mathrm{H}$, $\left.\mathrm{OCH}_{3}\right), 4.16\left(\mathrm{q}, J=7 \mathrm{~Hz}, 2 \mathrm{H}, \mathrm{CH}_{2}\right), 6.79-7.80(\mathrm{~m}, 14 \mathrm{H}, \mathrm{Ar}-\mathrm{H}), 8.05(\mathrm{~s}, 1 \mathrm{H}$, pyrazole H); MS m/z (\%) $531\left(\mathrm{M}^{+}+1,3\right), 530\left(\mathrm{M}^{+}, 4\right), 457(30), 388$ (29), 341 (13), 92 (24), 77 (100). Anal. Calcd. for $\mathrm{C}_{31} \mathrm{H}_{26} \mathrm{~N}_{6} \mathrm{O}_{3}$ (530.59): C, 70.18; H, 4.94; N, 15.84. Found: C, 70.08; H, 4.88; N, 15.77\%.

7-Methyl-2-(4-methoxyphenyl)-3-phenyl-4,5-dihydro-2H-pyrazolo[3,4-d]-pyridazin-4-one (11). White solid, (80\% yield), mp $266^{\circ} \mathrm{C}$; IR (KBr) $v_{\max } 3177(\mathrm{NH}), 1658(\mathrm{C}=\mathrm{O}) \mathrm{cm}^{-1} ;{ }^{1} \mathrm{H}$ NMR $\left(\mathrm{DMSO}-d_{6}\right) \delta 2.60\left(\mathrm{~s}, 3 \mathrm{H}, \mathrm{CH}_{3}\right), 3.83\left(\mathrm{~s}, 3 \mathrm{H}, \mathrm{OCH}_{3}\right), 6.88(\mathrm{~d}, J=9 \mathrm{~Hz}, 2 \mathrm{H}, \mathrm{ArH}), 7.24(\mathrm{~d}, J=$ 9Hz, 2H, ArH), 7.35-7.48 (m, 5H, Ar-H), 9.79 (s, 1H, NH); MS m/z (\%) $333\left(\mathrm{M}^{+}+1,9\right), 332$ $\left(\mathrm{M}^{+}, 52\right), 331$ (100), 115 (28), 107 (15), 89 (15), 77 (64). Anal. Calcd. for $\mathrm{C}_{19} \mathrm{H}_{16} \mathrm{~N}_{4} \mathrm{O}_{2}(332.36)$ : C, 68.66; H, 4.85; N, 16.86. Found: C, 68.53; H, 4.72; N, 16.71\%.

\section{Synthesis of compound 12}

A mixture of compound $11(0.66 \mathrm{~g}, 2 \mathrm{mmol})$ and dimethylformamide-dimethylacetal (DMFDMA) (2 g) was refluxed for $50 \mathrm{~h}$ then left to cool. Methanol was added to the cold mixture. The resulting solid was collected by filtration, washed with methanol, dried and finally crystallized from ethanol to afford the enaminone 12 as yellow solid, $\left(85 \%\right.$ yield), mp $230^{\circ} \mathrm{C}$; IR ( $\left.\mathrm{KBr}\right) v_{\max }$ $3177(\mathrm{NH}), 1658(\mathrm{C}=\mathrm{O}) \mathrm{cm}^{-1} ;{ }^{1} \mathrm{H} \mathrm{NMR}\left(\mathrm{CDCl}_{3}\right) \delta 2.87\left(\mathrm{~s}, 3 \mathrm{H}, \mathrm{CH}_{3}\right), 3.14\left(\mathrm{~s}, 3 \mathrm{H}, \mathrm{CH}_{3}\right), 3.29(\mathrm{~s}$, $\left.3 \mathrm{H}, \mathrm{OCH}_{3}\right), 5.60(\mathrm{~d}, J=13 \mathrm{~Hz}, 1 \mathrm{H}, \mathrm{N}=\mathrm{C}-\mathrm{CH}-), 7.25-7.48(\mathrm{~m}, 9 \mathrm{H}, \mathrm{Ar}-\mathrm{H}), 7.68(\mathrm{~d}, J=13 \mathrm{~Hz}, 1 \mathrm{H}$, =CH-N-), 11.93 (s, 1H, NH); MS m/z (\%): 387 (M , 7), 331 (100), 273 (11), 151 (35), 77 (28). Anal. Calcd. for $\mathrm{C}_{22} \mathrm{H}_{21} \mathrm{~N}_{5} \mathrm{O}_{2}$ (387.44): C, 68.20; H, 5.46; N, 18.08. Found: C, 68.29; H, 5.42; N, $18.00 \%$.

Synthesis of 7-[(3-Acetyl-1-phenyl-pyrazol-4-yl)]-2-(p-methoxyphenyl)-3-phenyl-4,5dihydro-2H-pyrazolo[3,4-d]pyridazin-4-one (10). To a stirred solution of enamine $12(0.39 \mathrm{~g}, 1$ $\mathrm{mmol})$ and the hydrazonoyl chloride $2 \mathrm{c}(0.196 \mathrm{~g}, 1 \mathrm{mmol})$ in dry dioxane $(20 \mathrm{~mL})$, triethylamine $(0.2 \mathrm{~mL})$ was added and the mixture was refluxed for $20 \mathrm{~h}$. The precipitated triethylamine hydrochloride was filtered off, and the filtrate was evaporated under reduced pressure. The residue was triturated with methanol. The solid product, so formed in each case, was collected by filtration, washed with water, dried, and crystallized from ethanol to give compound $\mathbf{1 0}$ as pale yellow solid, (70\% yield), mp $120^{\circ} \mathrm{C}$; IR ( KBr) $v_{\max } 3188(\mathrm{NH}), 1672,1665(\mathrm{C}=\mathrm{O}) \mathrm{cm}^{-1} ;{ }^{1} \mathrm{H}$ $\operatorname{NMR}\left(\mathrm{CDCl}_{3}\right) \delta 2.54\left(\mathrm{~s}, 3 \mathrm{H}, \mathrm{CH}_{3}\right), 3.82\left(\mathrm{~s}, 3 \mathrm{H}, \mathrm{OCH}_{3}\right), 7.05-7.44(\mathrm{~m}, 14 \mathrm{H}, \mathrm{Ar}-\mathrm{H}), 8.05(\mathrm{~s}, 1 \mathrm{H}$, pyrazole-H), 12.00 (s, 1H, NH); MS m/z (\%): 502 (M+, 2), 446 (3), 331 (37), 210 (11), 118 (24), 
77 (100). Anal. Calcd. for $\mathrm{C}_{29} \mathrm{H}_{22} \mathrm{~N}_{6} \mathrm{O}_{3}$ (502.54): C, 69.31; H, 4.41; N, 16.72. Found: C, 69.20; H, 4.40; N, 16.81\%.

\section{Pharmacology}

\section{Cytotoxic activity against human breast cancer (MCF-7) in vitro.}

The method applied is similar to that reported by Skehan et $\mathrm{al}^{37}$ using Sulfo-Rhodamine-B stain (SRB). Cells were plated in 96-multiwill plate $\left(10^{4}\right.$ cells/well) for $24 \mathrm{~h}$ before treatment with the test compound to allow attachment of cell to the wall of the plate, five different concentrations of the compound 4 under test $(0,5,12.5,25$, and $50 \mu \mathrm{g} / \mathrm{ml})$ were added to the cell monolayer in triplicate wells individual dose, monolayer cells were incubated with the compounds for $48 \mathrm{~h}$ at $37^{\circ} \mathrm{C}$ and in atmosphere of $5 \% \mathrm{CO}_{2}$. After $48 \mathrm{~h}$, cells were fixed, washed and stained with SRB stain, excess stain was washed with acetic acid and attached stain was recovered with tris-EDTA buffer, color intensity was measured in an ELISA reader, the relation between surviving fraction and drug concentration is plotted to get the survival curve of tumor cell line and the $\mathrm{IC}_{50}$ was calculated. The results are summarized in Table 1.

\section{References}

1. Shawali, A. S.; Parkanyi C., J. Heterocycl. Chem. 1980, 17, 833.

2. Shawali, A. S. Heterocycles 1983, 20, 2239.

3. Shawali, A. S. Chem. Rev. 1993, 93, 2731.

4. Shawali, A. S.; Abdallah, M. A. Adv. Heterocycl. Chem. 1995, 63, 277.

5. Shawali, A. S.; Elsheikh, S. M. J. Heterocycl. Chem. 2001, 38, 541.

6. Shawali, A. S.; Mosselhi, M. A. N. J. Heterocycl. Chem. 2003, 40, 1725.

7. Shawali, A. S.; Mosselhi, M. A. N. J. Sulfur. Chem. 2005, 26, 267.

8. Shawali, A. S.; Sherif, S. M. Current Org. Chem. 2007, 11, 773.

9. Shawali, A. S.; Edrees, M. M. Arkivoc 2007, ix, 293.

10. Shawali, A. S.; Gomha, S. M. Tetrahedron 2002, 58, 8559.

11. Greenhill, J. V. Chem. Soc. Rev. 1977, 6, 277.

12. Negri, G.; Kascheres, C.; Kascheres, A. J. J. Heterocycl. Chem. 2004, 41, 461.

13. Venkov, A. P.; Angelov, P. A. Synthesis 2003, 2221.

14. Michael, J. P.; de Koning, C. B.; Gravestock, D.; Hosken, G. D.; Howard, A. S.; Jungmann, C. M.; Krause, R. W. M.; Parsons, A. S.; Pelly, S. C.; Stanbury, T. V. Pure Appl. Chem. 1999, 71, 979.

15. Riyadh S. M.; Abdelhamid I. A.; Al-Matar H. M.; Hilmy N. M.; Elnagdi M. H. Heterocycles 2008, 75, 1849.

16. Palmieri G.; Cimarelli C. Arkivoc 2006, vi, 104.

17. Svete J. Arkivoc 2006, vii, 35.

18. Elassar, A.-Z. A.; El-Khair, A. A. Tetrahedron 2003, 59, 8463. 
19. Kascheres C. M. J. Braz. Chem. Soc. 2003, 14, 945.

20. Lui P.; Greenhill J. V. Adv. Heterocycl. Chem. 1997, 67, 207.

21. Erian, A. W. Chem. Rev. 1993, 93, 1991.

22. Wamhoff, H. Adv. Heterocyclic Chem. 1985, 38, 357.

23. Cuccovia, A. M.; Quina, F. H.; Chaimovich, H. Tetrahedron 1982, 38, 1972.

24. Albert, A. Adv. Heterocyclic Chem. 1982, 32, 1.

25. Hosti, L.; Schenone, P.; Menozzi G. J. Heterocycl. Chem. 1980, 17, 61.

26. Taylor, E. C. and McKillop, M. The Chemistry of Cyclic Enaminonitriles and orthoAminonitriles, Wiley, New York 1970, p. 306.

27. Blaha, K.; Cervinka O. Adv. Org. Chem. 1963, 4, 1.

28. Edafiogho I.; Kombian S. B.; Ananthalakshmi K. V. V.; Salamma N. N.; Eddington N. D.; Wilson T. L.; Alexander M. S.; Jackson P. L.; Hanson C. D.; Scott K. R. J. Pharm. Sci. 2007, 96, 2509.

29. Gaber H. M.; Bagley M. C. Chem. Med. Chem. 2009, 1.

30. Ibrahim M. K. A.; El-Ghandour A. H. H.; Abou-hadeed K.; Abdelhafiz I. S. J. Indian Chem. Soc. 1992, 69, 378.

31. Albar H. A.; Asiri A. M.; Faidallah H. M.; Abdullah M. A. J. Saudi Chem. Soc. 2001, 5, 353.

32. Dawood K. M. J. Heterocycl.Chem., 2005, 42, 221.

33. Komarova E. S.; Makarov V. A.; Alekseeva G. V.; Granik V. G. Russian Chem. Bull. Int. Ed. 2006, 55, 735 .

34. He F. Q.; Liu X. H.; Wang B. L.; Li Z. M. Heteroatom Chem. 2008, 19, 21.

35. Amer F. A.; Hammouda M.; El-Ahl A. S.; Abdelwahab B. F. J. Chin. Chem. Soc. 2007,54, 1543.

36. Eweiss N. F.; Osman A. O. J. Heterocycl. Chem. 1980, 17, 1713.

37. Skehan P.; Storeng R., J. Nat. Cancer Inst. 1990, 82, 1107 